INVITED COMMENTARY

\title{
Growth factors and human pituitary adenomas
}

\author{
Anna Spada \\ Institute of Endocrine Sciences, University of Milano, Ospedale Maggiore IRCCS, via F. Sforza 35, 20122 Milano, Italy
}

In recent years, the demonstration that human pituitary adenomas are monoclonal provides further support for the existence of genomic mutations occurring in the progenitor cell that subsequently undergoes clonal expansion. All the molecules that physiologically control pituitary cell differentiation and growth may be potentially oncogenic as they may become the target of mutations or be altered in their expression level. Since hypothalamic neuropeptides, transcription factors, receptors, peripheral target hormones and locally produced proteins are involved in ultimate pituitary development, all these proteins may be regarded as good candidates for participating in pituitary tumor induction and/or promotion. However, up to now the only mutations identified in a significant proportion of pituitary tumors occur in the Gs $\alpha$ gene and cause constitutive activation of the cAMP-dependent pathway (1).

Van Wijk et al. (2), in this issue of European Journal of Endocrinology, examine the effects of hypophysiotropic hormones on hormone secretion and cell proliferation in normal and tumoral canine corticotrophs in culture. They report that hypothalamic corticotropin-releasing hormone (CRH) and arginine vasopressin (AVP) do not exert any proliferative action on normal and tumoral cells, consistent with the idea that hyperstimulation of corticotrophs by CRH does not play any major role in the pathogenesis of Cushing's disease. They also report that insulin-like growth factor-I (IGF-I), a growth factor that increases the replication of corticotroph AtT-20 cells, is not mitogenic for either normal or tumoral corticotrophs. By contrast, tumoral cells replicate in response to an unknown agent present in fetal calf serum, presumably a growth factor different from IGF-I.

The pituitary is the source of several growth factors that control gene expression and pituitary cell commitment, migration and proliferation by an autocrine/ paracrine mechanism (3). This autocrine network consists of both growth factors and cytokines that are expressed directly on the pituitary cell membrane or released in the extracellular matrix. The major source of these peptides is the differentiated endocrine cell while the paracrine role of other cell types, such as folliculostellate cells, is controversial, particularly in man. Both growth factors and cytokines act through transmembrane receptors that consist of distinct subunits. Moreover, the same receptor or subunit may be shared by different ligands. Growth factor receptors generally possess intrinsic tyrosine kinase activity while cytokine signaling involves the activation of the JAK/STAT pathway. By alternative splicing soluble forms of growth factor and cytokine receptors may be secreted, serving as either carrier proteins or natural receptor antagonists.

Overproduction of autocrine mitogenic factors as well as mutations that constitutively activate growth factor signaling pathways may result in uncontrolled pituitary growth. Similarly, escape from inhibitory influences exerted by growth factors with antiproliferative properties may produce an activated pituitary phenotype. Among the classical growth factors, particular attention has been paid in recent years to the pituitary expression and action of factors belonging to the epidermal growth factor (EGF), transforming growth factor- $\alpha(\mathrm{TGF} \alpha)$ and $-\beta$ (TGF $\beta)$, fibroblast growth factor (FGF), nerve growth factor (NGF), and insulin-like growth factor (IGF) families. EGF has been detected in the anterior pituitary, particularly in gonadotrophs and somatotrophs, while EGF receptors (EGF-R) have been found in all subsets of pituitary cells. EGF potently stimulates prolactin (PRL) and adrenocorticotropin (ACTH) secretion by activating PRL and pro-opiomelanocortin (POMC) gene transcription. Depending on the different cell lines employed, EGF causes either an enhanced or an attenuated pituitary cell proliferation. In particular, EGF seems to promote the proliferation of corticotrophs and the differentiation of lactotrophs. Several lines of evidence indicate that in the majority of human functioning and nonfunctioning pituitary adenomas both EGF and EGF-R are expressed at a variable level, EGF-R being overexpressed in the most aggressive and recurrent adenomas. This observation is consistent with the notion that overexpression of the EGF-R structurally related protein c-erb2 results in cell transformation and tumorigenesis in nude mice. Indeed, there is a redundancy of ligands for the EGF-R in the pituitary. In fact, TGF $\alpha$ which shares the same receptor with EGF is present in the pituitary, predominantly in lactotrophs. The expression of these growth factors is differentially regulated, estrogens and protein kinase $\mathrm{C}$ being strong activators of TGF $\alpha$ expression. While EGF exerts a major role in lactotroph differentiation, $\mathrm{TGF} \alpha$ seems to be mitogenic for lactotrophs (3). In fact, it has been reported that targeting $\mathrm{TGF} \alpha$ expression to pituitary lactotrophs results in the development of PRL secreting tumors in the transgenic mice without changes in the proliferation of the other cell types (4). Moreover, it has been shown that rat lactotroph proliferation induced by estrogen treatment is 
preceded by an increase in TGF $\alpha$ mRNA level, the opposite occurring during bromocriptine treatment (3). The majority of human functioning and nonfunctioning pituitary tumors express TGF $\alpha$ together with EGF-R.

Basic FGF (bFGF) is a potent mitogenic and angiogenic factor that was first identified in the pituitary. This factor appears to be diffusely expressed in pituitary cells that possess both low- and high-affinity bFGF receptors (bFGF-R). Within the normal rat pituitary bFGF stimulates PRL, growth hormone $(\mathrm{GH})$ and thyrotropin (TSH) secretion, with a major effect on PRL synthesis and secretion. Although there are several indications that bFGF may be implicated in pituitary microangiogenesis, embryogenesis and differentiation, data on the mitogenic activity of bFGF in pituitary cells, particularly lactotrophs, are controversial (3). Consistent with the abundant presence of bFGF in the pituitary, both bFGF and bFGF-R appear to be expressed in human pituitary adenomas at a variable level. However, it remains to be established if there is a correlation between the expression of this growth factor and the clinical and morphological phenotype of aggressive pituitary tumors. Similarly, the pathological significance of the high serum levels of bFGF-like proteins of pituitary origin that may be observed in patients with multiple endocrine neoplasia type-1 (MEN-1) and sporadic pituitary tumors remains elusive. Other members of the FGF family have been hypothesized to play a role in pituitary tumorigenesis. FGF-4, the product of the hst gene that is located in close proximity to the MEN-1 locus, is a potent in vivo and in vitro mitogen for lactotrophs that is only expressed in the embryo and during adult life in neoplastic tissues. In human aggressive prolactinomas $h s t$ gene sequences possessing transforming activity when transfected into fibroblast NIH3T3 cells have been identified (5). In addition to FGF, a family of direct-acting endothelial cell mitogens and angiogenic factors referred to as vascular endothelial growth factors (VEGF) has been identified in the pituitary, mainly in the folliculostellate cells (6). Whether VEGF plays a role in the proliferation of blood vessels that characterizes estrogen-induced lactotroph tumors in the rat as well as some human prolactinomas remains to be established.

Although originally identified as a neuronal differentiation factor, NGF is also expressed in the pituitary. NGF seems to be mainly produced by somatotrophs, lactotrophs and thyrotrophs that also express the specific NGF receptor trkA. Several experimental models indicate a permissive role for NGF in promoting development and maintenance of the lactotroph phenotype. In particular, NGF cooperates with EGF and bFGF in directing differentiation of lactotrophs by stimulating PRL synthesis and D2 dopaminergic receptor expression (7). Moreover, the observation that mice overexpressing a pituitary-directed NGF transgene develop lactotroph hyperplasia suggest a proliferative action of this growth factor. A disruption of the NGF-mediated autocrine loop has been proposed to play a role in the development and progression of human prolactinomas (7). In fact, contrary to what has been observed in other pituitary tumors, prolactinomas removed from patients resistant to bromocriptine treatment seem to be unable to produce and release NGF in vitro. Interestingly, it has been proposed that exposure of the resistant prolactinomas to NGF results in their differentiation into a less aggressive, differentiated phenotype re-expressing D2 dopamine receptors (7).

IGF-I is expressed in pituitary interstitial cells while its specific receptor, together with the receptor for IGF-II and insulin, are expressed in endocrine pituitary cells. Pituitary IGF-I is involved in the reduction of GH mRNA levels and $\mathrm{GH}$ secretion while this factor plays a stimulatory role on the proliferation of cell lines, such as corticotroph AtT-20 cells (8). Human pituitary adenomas of different types contain IGF-I together with the IGF receptor (IGF-R), which in the somatotroph tumor appears to be intact (3).

While growth factors such as EGF, FGF and NGF are involved in the maintenance of the differentiated lactotroph phenotype, TFG $\beta$ plays an inhibitory action on lactotroph proliferation and PRL secretion. TGF $\beta$, particularly the TGF $\beta$ I isoform, is expressed in the pituitary with a preferential localization in gonadotrophs and lactotrophs. TGF $\beta$ transduces the signals by activating a series of membrane-bound and soluble receptor types that may also bind activin and inhibin. The antiproliferative action of TGF $\beta$ is mediated by several intracellular signals, involving the induction of the retinoblastoma suppressor gene and that of different cyclin-dependent kinase inhibitors as well as the inhibition of N-myc expression (9). While several pituitary tumor cell lines lack TGF $\beta$ expression and are unresponsive to this agent, TGF $\beta$ receptors (TGF $\beta-\mathrm{R})$ are detectable in human pituitary adenomas and presumably mediate the action of activin, which is expressed in pituitary gonadotrophs.

In addition to classical growth factors, several lines of evidence support the notion that cytokines modulate growth and differentiation of different cell types, including pituitary cells. In particular, cytokines seem to be involved in the regulation of the hypothalamicpituitary-adrenocortical function. Several interleukins (IL), particularly IL-1, IL-2 and IL-6, are expressed in the pituitary and exert hormonal and proliferative actions, particularly in corticotrophs (3). While IL-1 and IL-6 activate POMC gene expression and ACTH secretion mainly stimulating $\mathrm{CRH}$ release at the hypothalamic level, IL-2 seems directly to increase POMC mRNA and ACTH release from corticotrophs. The effect of ILs, particularly IL-6, on cell proliferation is controversial since either an increased or a reduced rate of cell division has been observed, depending on the different cell lines used. Human pituitary adenomas express the naturally occurring IL-1 receptor antagonist, suggesting a role of the IL-1 network in these tumors (10). Moreover, pituitary adenomas may produce and release IL-2 and IL-6, IL-2 in particular being present in ACTH-secreting adenomas (11). 


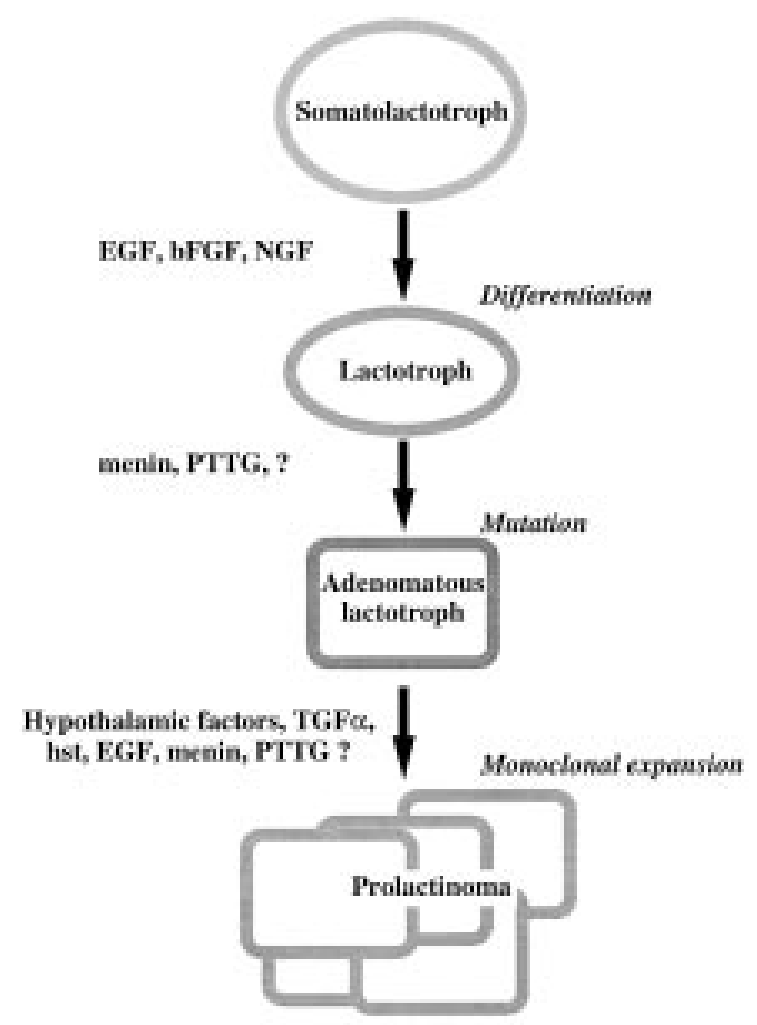

Figure 1 Multistep mechanisms of pituitary tumorigenesis: the prolactinoma example. Several pituitary growth factors direct differentiation of lactotrophs by an autocrine/paracrine mechanism (e.g. EGF, bFGF, NGF). Although the intrinsic defect leading to lactotroph transformation remains elusive, gain of function may result from either activation of protooncogenes (e.g. mutations in the pituitary-transforming gene, PTTG) or inactivation of tumor suppressor genes (e.g. menin gene in the 11 q13 region). Subsequently, promoting factors (hypothalamic factors and pituitary growth factors) are most likely required for clonal expansion of the transformed lactotroph.

Leukemia-inhibitory factor (LIF), which was originally isolated as an antiproliferative agent in a monocytic leukemia cell line, is expressed in the pituitary, particularly in corticotrophs and somatotrophs, and in human pituitary adenomas (3). Its action is mainly exerted in corticotrophs where it induces stimulation of POMC gene transcription and ACTH release as well as inhibition of cell proliferation (12).

It is clear from our current knowledge that the pituitary expresses a complex network of proteins that may have mitogenic, differentiative or antiproliferative properties. Although mutations of genes encoding growth factors and their receptors have not been identified in pituitary adenomas, modifications of the expression levels of these factors may have an important role in promoting the growth of already transformed pituitary cell clones and the expansion of small adenomas into large or invasive tumors (Fig. 1). Indeed, overexpression of factors possessing mitogenic activity when tested in tumoral cell lines or nude mice, such as TGF $\alpha$ and FGF, has been reported to occur in aggressive prolactinomas (3). Conversely, there is little evidence to support the hypothesis that lack of antiproliferative agents, such as TGF $\beta$ and LIF, may be involved in the promotion of pituitary neoplasia. Similarly, the impact of a defective expression of agents with differentiative properties on pituitary adenoma growth, such as NGF defect in lactotrophs, remains to be further elucidated. However, the wide spectrum of growth factors produced and released by the normal and tumoral pituitary strongly suggests that some of the agents described above may play a role in human adenoma progression, although direct demonstration of a causal relationship between growth factor expression and pituitary tumor growth is still lacking.

\section{References}

1 Landis CA, Masters SB, Spada A, Pace AM, Bourne HR \& Vallar L. GTPase inhibiting mutations activate the $\alpha$ chain of Gs and stimulate adenylyl cyclase in human pituitary tumours. Nature $1989340692-696$.

2 van Wijk PA, Rijnberk A, Croughs RJM, Meij BP \& Mol JA. Effects of corticotrophin-releasing hormone, vasopressin and insulin-like growth factor-I on proliferation of and adrenocorticotrophic hormone secretion by canine corticotrophic adenoma cells in vitro. European Journal of Endocrinology 1998138 309-315.

3 Ray D \& Melmed S. Pituitary cytokine and growth factor expression and action. Endocrine Review 199718 206-228.

4 McAndrew J, Paterson AJ, Asa SL, McCarthy KJ \& Kudlow JE. Targeting of transforming growth factor- $\alpha$ expression to pituitary lactotrophs in transgenic mice results in selective lactotroph proliferation and adenomas. Endocrinology $19951364479-4488$.

5 Gonsky R, Herman V, Melmed S \& Fagin J. Transforming DNA sequences present in human prolactin-secreting pituitary tumours. Molecular Endocrinology 19915 1687-1695.

6 Ferrara N, Houck K, Jakeman L \& Leung DW. Molecular and biological properties of the vascular endothelial growth factor family of proteins. Endocrine Review 199213 18-32.

7 Missale C, Boroni F, Losa M, Giovanelli M, Zanellato A, Dal Toso R, Balsari A \& Spano PF. Nerve growth factor suppresses the transforming phenotype of human prolactinomas. Proceedings of the National Academy of Sciences of the USA $1993907961-7965$.

8 Van Wijk PA, Neck JW van, Rijnberk A, Croughs RJM \& Mol JA. Proliferation of the murine corticotropic tumour cell line AtT20 is affected by hypophysiotropic hormones, growth factors and glucocorticoids. Molecular and Cellular Endocrinology 1995111 13-19.

9 Serra R \& Moeses HL. Tumor suppressor genes in the TGF $\beta$ signaling pathway? Nature Medicine 19962 390-391.

10 Sauer J, Arzt E, Gumprecht H, Hopfner U \& Stalla GK. Expression of interleukin-1 receptor antagonist in human pituitary adenomas in vitro. Journal of Clinical Endocrinology and Metabolism 1994 79 1857-1863.

11 Jones TH, Daniels M, James RA, Justice SK, McCorkle R, Price A, Kendall-Taylor P \& Weetman AP. Production of bioactive and immunoreactive interleukin-6 (IL-6) and expression of IL-6 messenger ribonucleic acid by human pituitary adenomas. Journal of Clinical Endocrinology and Metabolism 199478 180-187.

12 Stefana B, Ray DW \& Melmed S. Leukemia inhibitory factor (LIF) induces differentiation of pituitary corticotroph function: a neuroendocrine phenotypic switch. Proceedings of the National Academy of Sciences of the USA 199693 12502-12506.

Received 28 November 1997

Accepted 1 December 1997 